Węgrzyniak Agata, Wokurka Wojciech, Dudek Iga, Piech Piotr. What are the newest outcomes about Legg-Calve-Perthes disease etiology after 110 years over its discovering? - literature review. Journal of Education, Health and Sport. 2021;11(9):396-400. eISSN 2391-8306. DOI http://dx.doi.org/10.12775/JEHS.2021.11.09.050

https://apcz.umk.pl/JEHS/article/view/JEHS.2021.11.09.050

https://zenodo.org/record/5519997

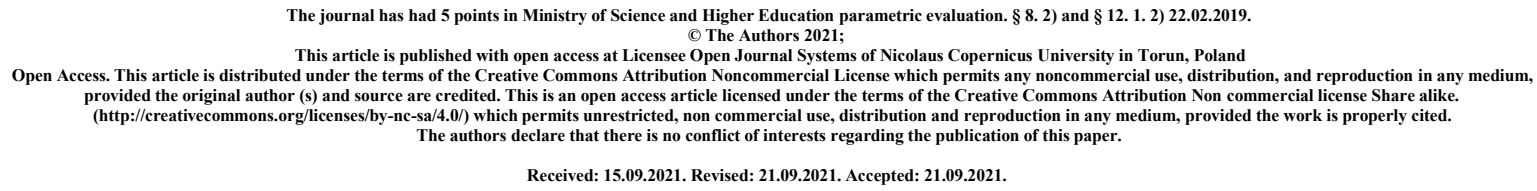

\title{
What are the newest outcomes about Legg-Calve-Perthes disease etiology after 110 years over its discovering? - literature review
}

\section{Agata Węgrzyniak ${ }^{1}$,Wojciech Wokurka ${ }^{1}$, Iga Dudek ${ }^{2}$ Supervisor: PhD Piotr Piech ${ }^{3,4}$}

Afiliacja:

${ }^{1}$ Human Anatomy Research Group, Department of Human Anatomy, Medical University of Lublin

2 Student Research Group at the Department of Epidemiology and Clinical Research Methodology, Medical University of Lublin

${ }^{3}$ Department of Human Anatomy, Medical University of Lublin

${ }^{4}$ Department of Orthopedics and Traumatology, Medical University of Lublin

ORCID ID:

Agata Węgrzyniak: https://orcid.org/0000-0001-8638-0013; agataw97@gmail.com

Wojciech Wokurka: https://orcid.org/0000-0001-9936-5656; wojciechwokurka@gmail.com

Iga Dudek: https://orcid.org/0000-0002-8101-074X; iga.dudek6@gmail.com

Piotr Piech: https://orcid.org/0000-0001-8717-7957 ; ppiotr.md@gmail.com

\begin{abstract}
Idiopathic avascular necrosis of the femoral head, known as Legg-Calve-Perthes disease (LCPD), is one of the most common necrosis among paediatric patients. Although it was described over 110 years ago its aetiology is still not established. Neither have effective treatment methods, which main aim is to maintain the joint with a full range of motion and to reduce the risk of developing degenerative changes. According to the literature, the exact aetiology of LCPD has still not been established. Increasingly, immunological and genetic disorders are highlighted in the literature, which may be an important cause of disease development. However, the number of reliable studies on this subject is very limited. Once
\end{abstract}


again, the influence of various risk factors present in fetal life has been analysed, but in a study with a significantly larger group of subjects. It has been repeatedly stressed that age, stage and radiological classification have a very important influence on the choice of treatment method and its effectiveness. Another important aspect is the need for reliable clinical trials to determine the effectiveness of bisphosphonate drugs and BMPs (bone morphogenetic proteins). In all studies, the need to perform more studies, on quantitatively larger groups of subjects, is significantly emphasized.

Key words: Legg-Calve-Perthes disease, Idiopathic avascular necrosis of the femoral head, etiology,

\section{Introduction}

Leg-Calve-Perthes disease is pediatric dysfunction that is described as aseptic avascular necrosis of proximal femur. Firstly this pathologic childhood condition was presented in 1910 by doctors after the disease has been named. However, since then aetiology and the most efficient treatment method are still unknown. The confirmed statement is that it occurs when the blood supply is temporarily disrupted which leads to the destruction of the epiphysis. Thereby as this state lasts weakened femoral head begins to collapse. Surprisingly this process is reversible and at one point blood supply comes back which results in both osteogenesis and femoral head rebuilding. Sadly all four states of LCPD; collapse, resorption, reossification and healing can last several years and according to literature in adulthood, it increase the probability of osteoarthritis of a hip. This disorder affects children between 2 and 14 years and the average age at 6.5 years. LCPD is more common among boys than girls and mostly unilateral (without significant differences in right and left hip involvement). This paper aims to present the latest information on Legg-Calve-Perthes disease, focusing on current reports about aetiology.

\section{Material and methods}

Basing on PubMed it was performed analyzation of databases aiming to find research and publications describing the newest outcomes about LCPD aetiology. To focus on the most 
recent works only works published after January 2016 were selected. As keywords were used: Legg-Calve-Perthes disease, aetiology.

\section{Results}

Using mentioned criteria and after preliminary analysis of abstracts, it was picked out 9 articles. All of them underwent full-text analysis.

\section{What is new in etiology}

\section{Risk factors during prenatal life}

It is underlined in the literature that some disturbances during prenatal life may have an effect on the unborn skeletal system and be the baseline for developing LCPD [1,2]. However, nowadays only one factor which is tobacco has been confirmed as a factor increasing the possibility of LCPD occurrence [3]. Other factors that are associated with LCPD are low birth weight, intrauterine growth restriction (IUGR) but literature outcomes are unconfirmed [2].

\section{Genetic and immunological factors}

Outcomes from works with the low number of participants underline the role of mutations in the COL2A1 gene and polymorphism in the eNOS (nitric oxide synthase) gene $[1,4,5]$. Once a report about the COL2A1 gene, which providing instructions for making the element of type II collagen was confirmed in epidemiological work on the other hand it was questioned in the twin study. Also, connection with polymorphism in MTFR gene-encoded information about methylenetetrahydrofolate reductase was invalid [1]. Promising results come from researches on animal models. One describes the potential role of cytokine IL-6, which high level in both blood and synovial fluid was established among patients suffer from LCPD [1,6]. Another influence of NF- $\kappa \mathrm{B}$ (nuclear factor kappa-light-chain-enhancer of activated B cells), which is a protein complex controlling among others cytokine production and cell survival $[1,6]$. Both of these works underline the potential possibility of a new therapeutic method basing on mentioned disorders. [1,6]

\section{Anatomical and individual factors}

An established connection was associated with obesity [7]. A high level of leptin and another metabolic dysfunction common among obese patients can influence the blood supply of a femoral head and thereby its structure [7]. This disturbance and also mechanical consequences of obesity increase the risk of microdamage in the cartilage of the femoral head [7]. On the 
other hand, biomechanical conditions in a hip joint may affect the blood patency of the epiphyseal vessels, and thus the disorders of ossification and the formation of epiphyseal cartilage [8].

\section{Mechanical factors}

Still, the most probable theory about LCPD development is mechanical provoked ischemic necrosis [1,9]. Modelling studies confirming the role of repeated mechanical stress in LCPD development [1,9]. Also based on this theory researchers are enabled to explain why patients with ADHD (attention-deficit hyperactivity disorder) suffer from LCPD significantly more frequent compering to the control group $[1,9]$.

\section{Conclusion}

Despite the passage of so many years since LCPD was described, the aetiology of this disorder remains undetermined. At present, there is still the only speculation as to what might be the determinants and factors that increase the likelihood of this disorder occurring. Therefore, there is still a need for more research, especially on large groups of subjects.

\section{References}

1. Pavone V, Chisari E, Vescio A, Lizzio C, Sessa G, Testa G. Aetiology of Legg-CalvéPerthes disease: A systematic review. World J Orthop. 2019 Mar 18;10(3):145-165. doi: 10.5312/wjo.v10.i3.145. PMID: 30918798; PMCID: PMC6429000.

2. Lindblad M, Josefsson A, Bladh M, Sydsjö G, Johansson T. Risk factors during pregnancy and delivery for the development of Perthes' disease, a nationwide Swedish study of 2.1 million individuals. BMC Pregnancy Childbirth. 2020 Mar 30;20(1):192. doi: 10.1186/s12884-020-2849-7. PMID: 32228493; PMCID: PMC7106730.

3. Perry DC, Thomson C, Pope D, Bruce CE, Platt MJ. A case control study to determine the association between Perthes' disease and the recalled use of tobacco during pregnancy, and biological markers of current tobacco smoke exposure. Bone Joint J. 2017 Aug;99-B(8):1102-1108. doi: 10.1302/0301-620X.99B8.BJJ-2016-1282.R1. PMID: 28768789.

4. Lindblad M, Josefsson A, Bladh M, Sydsjö G, Johansson T. Risk factors during pregnancy and delivery for the development of Perthes' disease, a nationwide Swedish study of 2.1 million individuals. BMC Pregnancy Childbirth. 2020 Mar 30;20(1):192. doi: 10.1186/s12884-020-2849-7. PMID: 32228493; PMCID: PMC7106730.

5. Azarpira MR, Ghilian MM, Sobhan MR, Mahdinezhad-Yazdi M, Aghili K, Ahrar H, Neamatzadeh H. Association of eNOS 27-bp VNTR, 894G $>$ T and 786T $>\mathrm{C}$ polymorphisms with susceptibility to Legg-Calve-Perthes Disease in Iranian children. J Orthop. 2019 Feb 26;16(2):137-140. doi: 10.1016/j.jor.2019.02.024. PMID: 30890857 ; PMCID: PMC6403418. 
6. Kuroyanagi G, Adapala NS, Yamaguchi R, Kamiya N, Deng Z, Aruwajoye O, Kutschke M, Chen E, Jo C, Ren Y, Kim HKW. Interleukin-6 deletion stimulates revascularization and new bone formation following ischemic osteonecrosis in a murine model. Bone. 2018 Nov;116:221-231. doi: 10.1016/j.bone.2018.08.011. Epub 2018 Aug 17. PMID: 30125727.

7. Neal DC, Alford TH, Moualeu A, Jo CH, Herring JA, Kim HK. Prevalence of Obesity in Patients With Legg-Calvé-Perthes Disease. J Am Acad Orthop Surg. 2016 Sep;24(9):660-5. doi: 10.5435/JAAOS-D-16-00120. PMID: 27471901.

8. Pinheiro M, Dobson CA, Perry D, Fagan MJ. New insights into the biomechanics of Legg-Calvé-Perthes' disease: The Role of Epiphyseal Skeletal Immaturity in Vascular Obstruction. Bone Joint Res. 2018 Feb;7(2):148-156. doi: 10.1302/20463758.72.BJR-2017-0191.R1. PMID: 29437587; PMCID: PMC5895949.

9. Pinheiro M, Dobson CA, Perry D, Fagan MJ. New insights into the biomechanics of Legg-Calvé-Perthes' disease: The Role of Epiphyseal Skeletal Immaturity in Vascular Obstruction. Bone Joint Res. 2018 Feb;7(2):148-156. doi: 10.1302/20463758.72.BJR-2017-0191.R1. PMID: 29437587; PMCID: PMC5895949. 